\title{
Elliptic units of cyclic unramified extensions of complex quadratic fields
}

\author{
by \\ FARShid HaJir (Cambridge, Mass.)
}

1. Introduction. Let $K$ be an abelian unramified extension of degree $n$ over a complex quadratic field $k$ of class number $h$. Let $H$ be the class number of $K$. Siegel [4] has defined a group of "elliptic units" of $K$ whose index in the full group of units is $\frac{c H}{h / n}$ where $c$ is a product of high powers of 2,3 , and $h$. By enlarging the group of elliptic units, Kersey (see [2]) was able to reduce this index to $\frac{H}{h / n}$. (Note that $h / n$ divides $H$ because the Hilbert class field of $k$ is an abelian unramified extension of $K$ of degree $h / n$.) Since examples with $H=h / n$ do exist, this index, for a general complex quadratic field $k$, is in some sense best possible.

Since the elliptic units (when embedded in $\mathbb{C}$ ) are given as values of elliptic modular functions, they can be approximated to high precision with ease. This makes it possible to devise a highly efficient procedure, based on the above index formula, for computing $H$. From this point of view, there are two reasons why Kersey's units are more desirable than those of Siegel: first, with Siegel's units, determining the divisibility of $H$ by 2 and 3 involves more computation; second, Siegel's units are high powers and will therefore be numerically less manageable (their minimal polynomials have large height). However, Siegel's group has the advantage that it comes equipped with a minimal set of generators, whereas Kersey provides $n(n-1) / 2$ generators for his group, whose rank is $n-1$. From a computational point of view, the lack of a minimal set of generators far outweighs the advantages of Kersey's units.

In the case that $K / k$ is a cyclic extension, we are able to find $n-1$ elliptic units which, together with the roots of unity of $K$, generate a group of "minimal index."

TheOREM 1.1. Let $k$ be a complex quadratic field of class number $h, K$ a cyclic unramified extension of degree $n$ over $k$. Let $E_{K}$ and $\mu_{K}$ denote the group of all units and the roots of unity of $K$, respectively. Let $H$ be 
the class number of $K$. Then there exist units $\varepsilon_{1}, \ldots, \varepsilon_{n-1}$ in $E_{K}$ given as a root of unity times ratios of values of the Dedekind eta function which, together with $\mu_{K}$, generate a subgroup $\mathcal{E}$ of $E_{K}$ with index $\left[E_{K}: \mathcal{E}\right]=\frac{H}{h / n}$.

For more precise statements, see Theorems 7.4 and 8.4. The proof is given in a sequence of lemmas, and does not make use of Kersey's proof.

Under the assumptions: $n$ is an odd prime, and $\left|\mu_{K}\right|=2$, Hayashi [1] has derived this index formula via Schertz's work. In addition, Hayashi describes an effective procedure for computing the index $\left[E_{K}: \mathcal{E}\right]$. Part of this procedure involves finding the roots of $\Delta$-quotients which are elements of $K$ by trial and error. In the last section, we indicate how the reciprocity law of complex multiplication determines these roots easily and we even give a simple formula (due to Villegas) for them when the discriminant of $k$ is prime to 6 .

I would like to thank H. M. Stark for his guidance and encouragement during the preparation of this paper.

2. Analytic preliminaries. For an arbitrary number field $M$, with $r_{1}$ real and $r_{2}$ pairs of complex conjugate embeddings, let $\mathcal{O}_{M}, h_{M}, R_{M}$, and $w_{M}$ denote the ring of integers, class number, regulator, and number of roots of unity of $M$, respectively. For an ideal class $C$ of $M$, the partial zeta function is defined by $\zeta_{M}(s, C)=\sum_{\mathfrak{a} \subseteq \mathcal{O}_{M},[\mathfrak{a}]=C} \mathbb{N}(\mathfrak{a})^{-s}$ where $\mathfrak{a}$ is a nonzero integral ideal of $k,[\mathfrak{a}]$ is the ideal class of $\mathfrak{a}$, and $\mathbb{N}$ denotes the absolute norm; it has a meromorphic continuation to $\mathbb{C}$. For a character $\chi$ of the class group $C \ell(M)$, we define the L-function $L(s, \chi)=\sum_{C \in C \ell(M)} \chi(C) \zeta_{M}(s, C)$. Finally, for the trivial character $\chi_{1}, L\left(s, \chi_{1}\right)$ is the Dedekind zeta function of $M$, denoted by $\zeta_{M}(s)$. We will use the abbreviation $e(z)=\exp (2 \pi i z)$.

The work of Dirichlet and Dedekind on zeta functions culminated in the determination of the first non-zero term in the Taylor expansion of $\zeta_{M}(s, C)$ at $s=1$. Hecke's functional equation allows one to carry out the expansion at $s=0$ :

$$
\zeta_{M}(s, C)=-\frac{R_{M}}{w_{M}} s^{r_{1}+r_{2}-1}+O\left(s^{r_{1}+r_{2}}\right) .
$$

Since the leading term is independent of the ideal class $C$,

$$
\zeta_{M}(s)=-\frac{h_{M} R_{M}}{w_{M}} s^{r_{1}+r_{2}-1}+O\left(s^{r_{1}+r_{2}}\right) .
$$

Let $k$ be a complex quadratic field and $K$ an abelian unramified extension of degree $n$ over $k$. Let $h=h_{k}, w=w_{k}, H=h_{K}, W=w_{K}$, and $R=$ $R_{K}$. We assume $w=2$, which rules out only two complex quadratic fields, $\mathbb{Q}(\sqrt{-3})$ and $\mathbb{Q}(\sqrt{-4})$, both of which have class number 1 and therefore do not concern us here. For an ideal class $C$ of $k$, we introduce $\lambda(C)=\zeta_{k}^{\prime}(0, C)$ 
as it will play a fundamental role in all that is to follow. Its evaluation, due to Kronecker, will be recalled in Section 5 .

Let $F$ be the Hilbert class field of $k$, i.e. the maximal abelian unramified extension of $k$. We fix an embedding of $F$ in $\mathbb{C}$ and think of $k$ and $K$ as subfields of this particular subfield of $\mathbb{C}$. For a prime ideal $\mathfrak{p}$ of $k$, let $\sigma(\mathfrak{p})$ denote the corresponding Frobenius automorphism of $F / k$; this automorphism of $F$ depends only on $[\mathfrak{p}]$ and gives rise to an isomorphism $\sigma: C \ell(k) \rightarrow G a \ell(F / k)$. The group $G a \ell(F / K)$, when considered as a subgroup of $G a \ell(F / k)$, corresponds, via $\sigma^{-1}$, to a subgroup $S$ of $C \ell(k)$, so that $\sigma$ induces an isomorphism $C \ell(k) / S \rightarrow G a \ell(K / k)$. A character $\chi$ of $G a \ell(K / k)$, therefore, may be viewed as a character of $C \ell(k)$ which is trivial on $S$.

LEMMA 2.1. With the above notation,

$$
-\frac{H R}{W}=L\left(0, \chi_{1}\right) \prod_{\chi \neq \chi_{1}} L^{\prime}(0, \chi),
$$

where the product is over those non-trivial characters $\chi$ of $C \ell(k)$ which are trivial on $S$.

Proof. Class field theory furnishes the factorization of the Dedekind zeta function of $K$ into L-functions:

$$
\zeta_{K}(s)=\prod_{\chi} L(s, \chi)
$$

Here, and in all that is to follow, $\prod_{\chi}$ means the product over all $n$ characters of $C \ell(k)$ which are trivial on $S$. Applying (1) and (2) to $k$, and $K$, respectively, we obtain

$$
\zeta_{k}(s, C)=-\frac{1}{2}+\lambda(C) s+O\left(s^{2}\right)
$$

and

$$
\zeta_{K}(s)=-\frac{H R}{W} s^{n-1}+O\left(s^{n}\right) .
$$

Using (5) and the orthogonality relation of characters, we get an expansion for the L-functions of $k$ :

$$
L(s, \chi)= \begin{cases}-h / 2+O(s) & \text { if } \chi=\chi_{1} \\ \left(\sum_{C \in C \ell(k)} \chi(C) \lambda(C)\right) s+O\left(s^{2}\right) & \text { if } \chi \neq \chi_{1}\end{cases}
$$

Finally, if we use (6) and (7) to expand the right and left hand sides of (4) at $s=0$ and equate the coefficients of $s^{n-1}$, we arrive at the desired result.

3. Imprimitive L-functions. From now on, we assume that $K$ is a cyclic unramified extension of $k$. Unless the prefix "integral" is used, by "ideal" we shall always mean "fractional ideal." Recall that for an ideal $\mathfrak{a}$ of $k,[\mathfrak{a}]$ denotes the ideal class of $\mathfrak{a}$ in $C \ell(k)$, and let $\{\mathfrak{a}\}$ denote the image 
of $[\mathfrak{a}]$ in $C \ell(k) / S$. Since there are infinitely many prime ideals of degree one in every ideal class, we let $\mathfrak{p}$ be an unramified prime ideal of degree one and norm $p>3$ such that $\{\mathfrak{p}\}$ generates the cyclic group $C \ell(k) / S$.

At this point, it will be advantageous to modify the L-functions slightly so that they all have a simple zero at $s=0$, giving a more symmetric version of (3). In addition, the expression for the derivative of the L-functions at 0 coming from the Kronecker limit formula will be greatly simplified as a result of this modification. For any character $\chi$ of $C \ell(k)$, define the modified L-function by removing the Euler $\mathfrak{p}$-factor as follows:

$$
L(s, \chi, \mathfrak{p})=L(s, \chi)\left(1-\chi([\mathfrak{p}]) p^{-s}\right) .
$$

This is the imprimitive L-function attached to $\chi$ viewed as a ray class character modulo $\mathfrak{p}$.

LEMMA 3.1.

$$
-\frac{H R}{W} n \log p=\prod_{\chi} L^{\prime}(0, \chi, \mathfrak{p}) .
$$

Proof. It is clear that $L(0, \chi, \mathfrak{p})=0$ for all $\chi$, including $\chi_{1}$, and

$$
L^{\prime}(0, \chi, \mathfrak{p})= \begin{cases}L\left(0, \chi_{1}\right) \log p & \text { if } \chi=\chi_{1}, \\ L^{\prime}(0, \chi)(1-\chi([\mathfrak{p}])) & \text { if } \chi \neq \chi_{1}\end{cases}
$$

Taking the product of these L-values over those $\chi$ which are trivial on $S$ yields

$$
\prod_{\chi} L^{\prime}(0, \chi, \mathfrak{p})=\left\{L\left(0, \chi_{1}\right) \prod_{\chi \neq \chi_{1}} L^{\prime}(0, \chi)\right\} \log p \prod_{\chi \neq \chi_{1}}(1-\chi([\mathfrak{p}])) .
$$

Since the set of characters of $C \ell(k)$ which are trivial on $S$ coincides with the set of characters of $C \ell(k) / S$, a cyclic group of order $n$ with generator $\{\mathfrak{p}\}$, we easily evaluate $\prod_{\chi \neq \chi_{1}}(1-\chi([\mathfrak{p}]))=\prod_{j=1}^{n-1}(1-e(j / n))=n$. The equality we seek is then a consequence of Lemma 2.1.

4. The group determinant. In this section, we recall the group determinant formula of Dedekind-Frobenius and use it to transform the right hand side of (8) into the determinant of a matrix whose entries are defined in terms of $\lambda$; the nature of these entries will be investigated in the next section.

Let $g_{1}, \ldots, g_{n}$ be the elements of an abelian group $G$ of order $n$, and assign a complex number $\Lambda(g)$ to each $g \in G$. According to [4, p. 78], the group determinant formula of Dedekind-Frobenius is

$$
\prod_{\psi} \sum_{g \in G} \psi(g) \Lambda(g)=\operatorname{det}\left(\Lambda\left(g_{i}^{-1} g_{j}\right)\right)_{1 \leq i, j \leq n}
$$

where $\psi$ runs through all characters of $G$. 
In order to apply this to the group $C \ell(k) / S$, recall that $\lambda(C)=\zeta_{k}^{\prime}(0, C)$, and define

$$
\begin{gathered}
\lambda_{\mathfrak{p}}(C)=\lambda(C)-\lambda\left(C[\mathfrak{p}]^{-1}\right)-\frac{1}{2} \log p, \\
\Lambda(\{C\})=\sum_{C^{\prime} \in S} \lambda_{\mathfrak{p}}\left(C C^{\prime}\right) .
\end{gathered}
$$

For any integer $m$, define $C_{m}=[\mathfrak{p}]^{m}$, so that $\left\{C_{1}\right\}, \ldots,\left\{C_{n}\right\}$ are the $n$ elements of $C \ell(k) / S$.

LEMMA 4.1.

$$
-\frac{H R}{W} n \log p=\operatorname{det}\left(\Lambda\left(\left\{C_{i}^{-1} C_{j}\right\}\right)\right)_{1 \leq i, j \leq n} .
$$

Proof. The first step is to evaluate $L^{\prime}(0, \chi, \mathfrak{p})$ in terms of $\lambda$. Using the definition of $L(s, \chi)$, and expanding the Euler $\mathfrak{p}$-factor $1-\chi([\mathfrak{p}]) p^{-s}$ as $1-\chi([\mathfrak{p}])\left(1-s \log p+O\left(s^{2}\right)\right)$, the Taylor expansion of $L(s, \chi, \mathfrak{p})$ at $s=0$ may be written

$$
\begin{aligned}
& L(s, \chi, \mathfrak{p}) \\
& \quad=\sum_{C \in C \ell(k)} \chi(C)\left[\zeta_{k}(s, C)-\zeta_{k}\left(s, C[\mathfrak{p}]^{-1}\right)+s \zeta_{k}\left(s, C[\mathfrak{p}]^{-1}\right) \log p\right]+O\left(s^{2}\right) .
\end{aligned}
$$

Evaluating the derivative at $s=0$ is then a simple matter:

$$
L^{\prime}(0, \chi, \mathfrak{p})=\sum_{C \in C \ell(k)} \chi(C)\left[\zeta_{k}^{\prime}(0, C)-\zeta_{k}^{\prime}\left(0, C[\mathfrak{p}]^{-1}\right)+\zeta_{k}\left(0, C[\mathfrak{p}]^{-1}\right) \log p\right] .
$$

Since by $(5), \zeta_{k}\left(0, C^{\prime}\right)=-1 / 2$ for any class $C^{\prime} \in C \ell(k)$, we have

$$
\begin{aligned}
L^{\prime}(0, \chi, \mathfrak{p}) & =\sum_{C \in C \ell(k)} \chi(C)\left[\lambda(C)-\lambda\left(C[\mathfrak{p}]^{-1}\right)-\frac{1}{2} \log p\right] \\
& =\sum_{C \in C \ell(k)} \chi(C) \lambda_{\mathfrak{p}}(C) .
\end{aligned}
$$

For a character $\chi$ which is trivial on $S$, we may rewrite the above sum as a sum over $C \ell(k) / S$ as follows:

$$
\begin{aligned}
L^{\prime}(0, \chi, \mathfrak{p}) & =\sum_{\{C\} \in C \ell(k) / S} \chi(\{C\}) \sum_{C^{\prime} \in S} \lambda_{\mathfrak{p}}\left(C C^{\prime}\right) \\
& =\sum_{\{C\} \in C \ell(k) / S} \chi(\{C\}) \Lambda(\{C\}) .
\end{aligned}
$$

Lemma 3.1, when combined with (14) and (10), yields the result.

5. Kronecker's limit formula and complex multiplication. We now use Kronecker's first limit formula and some facts from the theory of complex multiplication to investigate $\Lambda\left(\left\{C_{i}^{-1} C_{j}\right\}\right)$. 
For an ideal $\mathfrak{b}$ of $k$, with $\mathbb{Z}$-basis $\left[\omega_{1}, \omega_{2}\right]$, satisfying $\Im\left(\omega_{1} / \omega_{2}\right)>0$, define

$$
\Delta(\mathfrak{b})=\left(\frac{2 \pi}{\omega_{2}}\right)^{12} \Delta\left(\frac{\omega_{1}}{\omega_{2}}\right),
$$

where

$$
\Delta(z)=\eta(z)^{24}, \quad \eta(z)=e(z / 24) \prod_{m \geq 1}(1-e(m z)) .
$$

It is well known that $\Delta$ does not vanish on the upper half plane and is invariant under the standard action of $S L_{2}(\mathbb{Z})$. Kronecker's first limit formula (see [4] or [5]) gives an expression for $\lambda(C)$ in terms of $\Delta$ :

$$
\lambda(C)=-\frac{1}{24} \log \left|\mathbb{N}(\mathfrak{b})^{6} \Delta(\mathfrak{b})\right|^{2}
$$

where $\mathfrak{b}$ is an ideal of $k$ in the class $C^{-1}$. By the multiplicativity of $\mathbb{N}$ and the invariance of $\Delta$ under the modular group, the limit formula is independent of the choice of the ideal $\mathfrak{b}$ in $C^{-1}$ and $\Delta(\mathfrak{b})$ is independent of the choice of its basis.

We apply the limit formula (15) to compute $\lambda_{\mathfrak{p}}(C)$ and $\Lambda(\{C\})$, defined in (11) and (12):

$$
\begin{aligned}
\lambda_{\mathfrak{p}}(C) & =-\frac{1}{24} \log \left|\mathbb{N}(\mathfrak{b})^{6} \Delta(\mathfrak{b})\right|^{2}+\frac{1}{24} \log \left|\mathbb{N}(\mathfrak{b} \mathfrak{p})^{6} \Delta(\mathfrak{b} \mathfrak{p})\right|^{2}-\frac{1}{24} \log \left|\mathbb{N}^{6}\right|^{2} \\
& =-\frac{1}{24} \log \left|\frac{\Delta(\mathfrak{b})}{\Delta(\mathfrak{b} \mathfrak{p})}\right|^{2}
\end{aligned}
$$

and

$$
\Lambda(\{C\})=\sum_{C^{\prime} \in S}-\frac{1}{24} \log \left|\frac{\Delta\left(\mathfrak{b} \mathfrak{b}^{\prime}\right)}{\Delta\left(\mathfrak{b} \mathfrak{b}^{\prime} \mathfrak{p}\right)}\right|^{2}=-\frac{1}{24} \log \left|\prod_{C^{\prime} \in S} \frac{\Delta\left(\mathfrak{b} \mathfrak{b}^{\prime}\right)}{\Delta\left(\mathfrak{b} \mathfrak{b}^{\prime} \mathfrak{p}\right)}\right|^{2},
$$

with $[\mathfrak{b}]=C^{-1}$ and $\left[\mathfrak{b}^{\prime}\right]=C^{-1}$.

We recall the following facts from the theory of complex multiplication $([5])$.

Lemma 5.1. For any pair of ideals $\mathfrak{m}, \mathfrak{n}$ of $k, \alpha=\Delta(\mathfrak{m}) / \Delta(\mathfrak{n}) \in F^{*}$ and generates the ideal $(\alpha) \mathcal{O}_{F}=(\mathfrak{n} / \mathfrak{m})^{12}$. The conjugates of $\alpha$ are given explicitly by the reciprocity law:

$$
\overline{\left(\frac{\Delta(\mathfrak{m})}{\Delta(\mathfrak{n})}\right)}=\frac{\Delta(\overline{\mathfrak{m}})}{\Delta(\overline{\mathfrak{n}})}, \quad\left(\frac{\Delta(\mathfrak{m})}{\Delta(\mathfrak{n})}\right)^{\sigma(\overline{\mathfrak{a}})}=\frac{\Delta(\mathfrak{m} \mathfrak{a})}{\Delta(\mathfrak{n} \mathfrak{a})}
$$

for any ideal $\mathfrak{a}$ of $k$.

We use the lemma to investigate the $\Delta$-quotient in (16):

$$
\frac{\Delta\left(\mathfrak{b} \mathfrak{b}^{\prime}\right)}{\Delta\left(\mathfrak{b} \mathfrak{b}^{\prime} \mathfrak{p}\right)}=\left(\frac{\Delta(\mathfrak{b})}{\Delta(\mathfrak{b} \mathfrak{p})}\right)^{\sigma\left(\overline{\overline{\mathfrak{b}}^{\prime}}\right)}=\left(\frac{\Delta(\mathfrak{b})}{\Delta(\mathfrak{b} \mathfrak{p})}\right)^{\sigma\left(C^{\prime}\right)}
$$


since $\left[\overline{\mathfrak{b}^{\prime}}\right]=\left[\mathfrak{b}^{\prime}\right]^{-1}=C^{\prime}$. Recalling that $\sigma$ maps $S$ isomorphically to $\operatorname{Ga\ell }(F / K)$, we recognize the expression appearing inside the absolute values in (16) as a relative norm from $F$ to $K$ :

$$
\begin{aligned}
\prod_{C^{\prime} \in S} \frac{\Delta\left(\mathfrak{b} \mathfrak{b}^{\prime}\right)}{\Delta\left(\mathfrak{b} \mathfrak{b}^{\prime} \mathfrak{p}\right)} & =\prod_{C^{\prime} \in S}\left(\frac{\Delta(\mathfrak{b})}{\Delta(\mathfrak{b} \mathfrak{p})}\right)^{\sigma\left(C^{\prime}\right)} \\
& =\prod_{\sigma \in G a \ell(F / K)}\left(\frac{\Delta(\mathfrak{b})}{\Delta(\mathfrak{b} \mathfrak{p})}\right)^{\sigma}=\mathbb{N}_{F / K}\left(\frac{\Delta(\mathfrak{b})}{\Delta(\mathfrak{b} \mathfrak{p})}\right)
\end{aligned}
$$

Therefore, whenever $[\mathfrak{b}]=C^{-1}$,

$$
\Lambda(\{C\})=-\frac{1}{24} \log \left|\mathbb{N}_{F / K}\left(\frac{\Delta(\mathfrak{b})}{\Delta(\mathfrak{b} \mathfrak{p})}\right)\right|^{2} .
$$

Recall that we have defined $C_{m}=[\mathfrak{p}]^{m}$ for any integer $m$. With the choice $\mathfrak{b}_{m}=\mathfrak{p}^{-m}$,

$$
\Lambda\left(\left\{C_{m}\right\}\right)=-\frac{1}{24} \log \left|\mathbb{N}_{F / K}\left(\frac{\Delta\left(\mathfrak{p}^{-m}\right)}{\Delta\left(\mathfrak{p}^{1-m}\right)}\right)\right|^{2} .
$$

Let $a_{i j}=\Lambda\left(\left\{C_{i}^{-1} C_{j}\right\}\right)$ for $1 \leq i, j \leq n$ and let $\mathcal{A}$ denote the $n \times n$ matrix whose entry in the $i$ th row and $j$ th column is $a_{i j}$. Since $\left\{C_{i}^{-1} C_{j}\right\}=\left\{C_{j-i}\right\}$,

$$
a_{i j}=-\frac{1}{24} \log |\alpha(i, j)|^{2} \text {, }
$$

where we have set

$$
\alpha(i, j)=\mathbb{N}_{F / K}\left(\frac{\Delta\left(\mathfrak{p}^{i-j}\right)}{\Delta\left(\mathfrak{p}^{i-j+1}\right)}\right) .
$$

Finally, we write (13) more simply as

$$
-\frac{H R}{W} n \log p=\operatorname{det} \mathcal{A} .
$$

6. Transforming $\mathcal{A}$ into a regulator matrix. For any number $\gamma \in K$, let $\gamma^{(1)}, \ldots, \gamma^{(n)}$ be its conjugates over $k$ in some fixed order. Suppose $\varepsilon_{1}, \ldots, \varepsilon_{n-1}$ are units of $K$. Let us call the $n-1 \times n-1$ matrix $\mathcal{R}\left(\varepsilon_{1}, \ldots, \varepsilon_{n-1}\right)=\mathcal{R}$ whose $i j$ th entry is $\log \left|\varepsilon_{i}^{(j)}\right|^{2}$, the "regulator matrix" of these units. The absolute value of its determinant is non-zero if and only if $\varepsilon_{1}, \ldots, \varepsilon_{n-1}$ generate, together with the roots of unity in $K$, a subgroup of $E_{K}$ of finite index, and moreover this index is then precisely the quotient $|\operatorname{det} \mathcal{R}| / R$ where $R$ is the regulator of $K$ [7, Lemma 4.15]. Therefore, to obtain an index formula involving $H$, we would like to transform (17) into $H R=(h / n)|\operatorname{det} \mathcal{R}|$ for some regulator matrix $\mathcal{R}$. The only tools we need to accomplish this are the complex multiplication lemma above and an algebraic lemma of Stark. 
Remarks. 1. By the complex multiplication lemma, for all $i, j$, $\Delta\left(\mathfrak{p}^{i-j}\right) / \Delta\left(\mathfrak{p}^{i-j+1}\right)$ generates the ideal $\mathfrak{p}^{12}$ in $F$, hence $\alpha(i, j)$ generates $\mathfrak{p}^{12 h / n}$ in $K$. Therefore, the quotient of any two $\alpha(i, j)$ 's is a unit of $K$.

2. For any $\gamma \in K$, let $\gamma^{(j)}=\gamma^{\sigma\left(C_{j}\right)}$ for $j=1, \ldots, n$. Then $\gamma^{(1)}, \ldots, \gamma^{(n)}$ are the conjugates of $\gamma$ over $k$ with $\gamma=\gamma^{(n)}$. Upon setting

$$
\alpha_{i}=\mathbb{N}_{F / K}\left(\frac{\Delta\left(\mathfrak{p}^{i}\right)}{\Delta\left(\mathfrak{p}^{i+1}\right)}\right),
$$

it follows from the complex multiplication lemma that $\alpha(i, j)=\alpha_{i}^{(j)}$ for $1 \leq i, j \leq n$.

As a result of Remark 2, it is clear that $\mathcal{A}$ would be a regulator matrix but for three flaws: first that the numbers inside the absolute value signs are not units, second that the dimension of $\mathcal{A}$ is $n$ not $n-1$, and finally that every entry has an extra factor of $-1 / 24$.

Remark 1 provides the inspiration for correcting the first of these flaws; we reduce the dimension via row and column operations; finally, in the next section, we use an algebraic lemma to take out the extra factors of $-1 / 24$.

Define, for $i=1, \ldots, n-1$,

$$
\beta_{i}=\frac{\alpha_{i}}{\alpha_{i+1}},
$$

and note that for $j=1, \ldots, n$,

$$
\beta_{i}^{(j)}=\frac{\alpha_{i}^{(j)}}{\alpha_{i+1}^{(j)}}=\frac{\alpha(i, j)}{\alpha(i+1, j)}
$$

is a unit of $K$ by Remark 1. Define the matrix

$$
\mathcal{B}=\left(-\frac{1}{24} \log \left|\beta_{i}^{(j)}\right|^{2}\right)_{1 \leq i, j \leq n-1} .
$$

LEMMA 6.1.

$$
\frac{W}{2} \frac{\operatorname{det} \mathcal{B}}{R}=\frac{H}{h / n} .
$$

Proof. Let $\mathcal{B}^{\prime}$ be the $n \times n$ matrix whose $i j$ th entry $b_{i j}$ is given by

$$
b_{i j}= \begin{cases}a_{i j}-a_{i+1, j} & \text { for } i=1, \ldots, n-1, \\ \sum_{l=1}^{n} a_{l j} & \text { for } i=n .\end{cases}
$$

Then it is an easy exercise in linear algebra to verify that

$$
\operatorname{det} \mathcal{B}^{\prime}=n \operatorname{det} \mathcal{A} \text {. }
$$

We now examine the matrix $\mathcal{B}^{\prime}$. In the first $n-1$ rows of $\mathcal{B}^{\prime}$, the entries are

$$
b_{i j}=-\frac{1}{24} \log |\alpha(i, j) / \alpha(i+1, j)|^{2}=-\frac{1}{24} \log \left|\beta_{i}^{(j)}\right|^{2} .
$$


On the other hand, recalling that $a_{i j}=\Lambda\left(\left\{C_{i}^{-1} C_{j}\right\}\right)$, we compute the last row of $\mathcal{B}^{\prime}$ :

$$
\begin{aligned}
b_{n j} & =\sum_{i=1}^{n} \Lambda\left(\left\{C_{i}^{-1} C_{j}\right\}\right)=\sum_{m=1}^{n} \Lambda\left(\left\{C_{m}\right\}\right) \\
& =L^{\prime}\left(0, \chi_{1}, \mathfrak{p}\right)=L\left(0, \chi_{1}\right) \log p=\frac{-h}{2} \log p
\end{aligned}
$$

the last three equalities being due in turn to (14), (9), and (7).

In short,

$$
\mathcal{B}^{\prime}=\left(\begin{array}{ccc}
-\frac{1}{24} \log \left|\beta_{1}^{(1)}\right|^{2} & \cdots & -\frac{1}{24} \log \left|\beta_{1}^{(n)}\right|^{2} \\
\vdots & & \vdots \\
-\frac{1}{24} \log \left|\beta_{n-1}^{(1)}\right|^{2} & \cdots & -\frac{1}{24} \log \left|\beta_{n-1}^{(n)}\right|^{2} \\
-\frac{h}{2} \log p & \cdots & -\frac{h}{2} \log p
\end{array}\right) .
$$

It is clear that $\mathcal{B}$ is the matrix that results by deleting the last row and last column of $\mathcal{B}^{\prime}$. Furthermore,

$$
\operatorname{det} \mathcal{B}^{\prime}=-\frac{h}{2} n \log p \operatorname{det} \mathcal{B} .
$$

To see this, we add the first $n-1$ columns of $\mathcal{B}^{\prime}$ to the last column, which then becomes

$$
\left(\begin{array}{c}
-\frac{1}{24} \log \left|\mathbb{N}_{K / k}\left(\beta_{1}\right)\right|^{2} \\
\vdots \\
-\frac{1}{24} \log \left|\mathbb{N}_{K / k}\left(\beta_{n-1}\right)\right|^{2} \\
-\frac{h}{2} n \log p
\end{array}\right)=\left(\begin{array}{c}
0 \\
\vdots \\
0 \\
-\frac{h}{2} n \log p
\end{array}\right)
$$

the last equality owing to the fact that $\mathbb{N}_{K / k}\left(\beta_{i}\right)$ is a unit of $k$ and hence has absolute value 1 . The lemma follows by combining (19) and (20) with (17).

7. Reducing the index. In order to eliminate the factors of $-1 / 24$ in $\mathcal{B}$, we now use an algebraic lemma of Stark to show that certain combinations of the $\beta_{i}$ are high powers in $K$.

LEMma 7.1. Let $m$ be a positive integer. Let $\widetilde{K} / k$ be an abelian extension with conductor $\mathfrak{f}$. Let $\widetilde{W}$ be the number of roots of unity in $\widetilde{K}$. Suppose that $\delta \neq 0$ is a number such that $\alpha=\delta^{m}$ is in $\widetilde{K}$ and $\widetilde{K}(\delta)$ is abelian over $k$. If $\mathfrak{q}$ is a prime ideal of $k$ of norm $q$ relatively prime to $m \alpha \mathfrak{f}$, then $\alpha^{q} / \alpha^{\tilde{\sigma}(\mathfrak{q})}$ and $\alpha^{(\widetilde{W}, m)}$ are $m$-th powers in $\widetilde{K}$ itself, where $\widetilde{\sigma}(\mathbf{q})$ denotes the Frobenius automorphism of $\mathfrak{q}$ in $\widetilde{K} / k$.

Proof. This is (a weak version of) [5, Lemma 6].

Lemma 7.2. For each $i, \beta_{i}^{W}$ is the 48 th power of a unit in $K$. 
Proof. The key fact (see [5]), is that when $\mathfrak{m}, \mathfrak{n}$ are ideals of $k$, adjoining any 24 th root of $\Delta(\mathfrak{m}) / \Delta(\mathfrak{n})$ to $K$ yields an abelian extension of $k$. Begin by observing from (18) and Lemma 5.1 that $\beta_{i}=\alpha_{i} / \alpha_{i}^{\sigma}$, where $\sigma=\sigma(\overline{\mathfrak{p}})$. By Lemma 7.1 (using $m=24$ ), $\alpha_{i}^{p} / \alpha_{i}^{\sigma}$ and $\alpha_{i}^{W}$ are 24 th powers in $K$. Since $p$ is odd and $W$ is even, $\left(\alpha_{i}^{p} / \alpha_{i}^{\sigma}\right)^{W}$ and $\alpha_{i}^{W(1-p)}$ are 48th powers. The lemma follows since $\beta_{i}^{W}$ is the product of these numbers.

Let $\varrho_{1}$ be a unit of $K$ such that $\varrho_{1}^{-48}=\beta_{1}^{W}$, and define for $i=2, \ldots, n-1$,

$$
\varrho_{i}=\varrho_{1}^{\sigma\left(C_{i-1}\right)},
$$

so that

$$
\varrho_{i}^{-48}=\beta_{i}^{W}
$$

(In the next section, we will write these units more explicitly.) Then

$$
\mathcal{B}=\left(\frac{2}{W} \log \left|\varrho_{i}^{(j)}\right|^{2}\right)_{1 \leq i, j \leq n-1} .
$$

Recall that $W$ is a divisor of 12 [2, p. 217]. We see immediately that if $W=2, \mathcal{B}=\mathcal{R}\left(\varrho_{1}, \ldots, \varrho_{n-1}\right)$ is a regulator matrix and hence by Lemma 6.1,

$$
\left[E_{K}:\left\langle-1, \varrho_{1}, \ldots, \varrho_{n-1}\right\rangle\right]=\frac{H}{h / n} .
$$

This completes the proof of Theorem 1.1 when $W=2$. If $W>2$, we must work a little harder.

Lemma 7.3. For each $i, \varrho_{i} / \varrho_{i+1}^{p}$ is a $W / 2$-power in $K$.

Proof. This is a direct consequence of Lemma 7.1 (with $m=W / 2, \mathfrak{q}=$ $\mathfrak{p}$ and $\left.\alpha=\varrho_{i+1}\right)$, together with the observation that $\varrho_{i+1}^{\sigma(\mathfrak{p})}=\varrho_{i}$.

Let $\gamma_{1}$ be some unit of $K$ satisfying $\gamma_{1}^{W / 2}=\varrho_{1} / \varrho_{2}^{p}$, and define for $i=$ $2, \ldots, n-2$,

$$
\gamma_{i}=\gamma_{1}^{\sigma\left(C_{i-1}\right)},
$$

so that

$$
\gamma_{i}^{W / 2}=\varrho_{i} / \varrho_{i+1}^{p} .
$$

Define units $\varepsilon_{1}, \ldots, \varepsilon_{n-1}$ of $K$ as follows:

$$
\varepsilon_{i}= \begin{cases}\gamma_{i} & \text { for } i=1, \ldots, n-2, \\ \varrho_{n-1} & \text { for } i=n-1 .\end{cases}
$$

THEOREM 7.4. Let $\mathcal{E}=\left\langle\mu_{K}, \varepsilon_{1}, \ldots, \varepsilon_{n-1}\right\rangle$ be the subgroup of $E_{K}$ generated by the elliptic units $\varepsilon_{1}, \ldots, \varepsilon_{n-1}$, together with the roots of unity of $K$. 
Note that when $W=2, \mathcal{E}=\left\langle-1, \varrho_{1}, \ldots, \varrho_{n-1}\right\rangle$. The index of this subgroup is

$$
\left[E_{K}: \mathcal{E}\right]=\frac{H}{h / n} .
$$

Pr o o f. Let $R_{1}, \ldots, R_{n-1}$ be the rows of $\mathcal{B}$. Let $\mathcal{R}^{\prime}$ be the $n-1 \times n-1$ matrix whose rows $R_{1}^{\prime}, \ldots, R_{n-1}^{\prime}$ are given by

$$
\begin{aligned}
& R_{1}^{\prime}=R_{1}-p R_{2}, \\
& R_{2}^{\prime}=R_{2}-p R_{3}, \\
& \vdots \\
& R_{n-2}^{\prime}=R_{n-2}-p R_{n-1}, \\
& R_{n-1}^{\prime}=R_{n-1} .
\end{aligned}
$$

Then $\operatorname{det} \mathcal{R}^{\prime}=\operatorname{det} \mathcal{B}$, and

Now define the regulator matrix

$$
\mathcal{R}^{\prime}=\left(\begin{array}{ccc}
\log \left|\gamma_{1}^{(1)}\right|^{2} & \cdots & \log \left|\gamma_{1}^{(n-1)}\right|^{2} \\
\vdots & & \vdots \\
\log \left|\gamma_{n-2}^{(1)}\right|^{2} & \ldots & \log \left|\gamma_{n-2}^{(n-1)}\right|^{2} \\
\frac{2}{W} \log \left|\varrho_{n-1}^{(1)}\right|^{2} & \cdots & \frac{2}{W} \log \left|\varrho_{n-1}^{(n-1)}\right|^{2}
\end{array}\right) .
$$

$$
\mathcal{R}=\left(\begin{array}{ccc}
\log \left|\gamma_{1}^{(1)}\right|^{2} & \cdots & \log \left|\gamma_{1}^{(n-1)}\right|^{2} \\
\vdots & & \vdots \\
\log \left|\gamma_{n-2}^{(1)}\right|^{2} & \cdots & \log \left|\gamma_{n-2}^{(n-1)}\right|^{2} \\
\log \left|\varrho_{n-1}^{(1)}\right|^{2} & \cdots & \log \left|\varrho_{n-1}^{(n-1)}\right|^{2}
\end{array}\right)
$$

so that by Lemma 6.1,

$$
\frac{H}{h / n}=\frac{\operatorname{det} \mathcal{R}}{R} .
$$

This completes the proof of the theorem.

8. An explicit 12th root of $\Delta(\mathfrak{m}) / \Delta(\mathfrak{n})$ in $F$. Recall that $F$, the Hilbert class field of $k$, is embedded in $\mathbb{C}$. The unit $\varrho_{1}$ was defined in the last section as a certain 48 th root of a $\Delta$-quotient. As is clear from the proof of Theorem 8.1 below, once it is known that some 48th root is in $K$, finding it takes only one application of the reciprocity law of complex multiplication, which, using Stark's formulation, is easy to carry out. Under the assumption that the discriminant of $k$ is prime to 6 , it is even possible to write down a simple, explicit formula for a 12 th root of $\Delta(\mathfrak{m}) / \Delta(\mathfrak{n})$ in $F$. This formula is due to F. R. Villegas [6], though the proof given here is different from his. I would like to express my thanks for his permission to include this formula 
here. Finally, G. Robert [3] has provided an alternate recipe for determining which roots of $\Delta$-quotients land in the Hilbert class field.

Throughout this section, the discriminant $-D$ of the complex quadratic field $k$ is assumed to be prime to 6 so that $w_{F}=2$. Let $\mathfrak{m}$ be any primitive (i.e. not divisible by an integer $>1$ ) integral ideal of $k$ of norm $m$ prime to $6 D$, with $\mathbb{Z}$-basis $[\omega-t, m]$ for some integer $t$ and $\omega=(1+\sqrt{-D}) / 2$. Let $\mathcal{O}=[\omega, 1]$ be the ring of integers of $k$. It will be convenient to define $e_{r}(s)=e(s / r)$.

Definition. For $\mathfrak{m}$ as above, set

$$
\eta(\mathfrak{m})=e_{24}(m(2-t)) \eta\left(\frac{t-\bar{\omega}}{m}\right) .
$$

Using the modular property: $\eta(z+1)=e_{24}(1) \eta(z)$, and the fact that $m^{2} \equiv 1(\bmod 24)$, it is easy to see that the above definition is independent of the choice of $t$.

TheOREM 8.1. With the above assumptions on $D$ and $\mathfrak{m}$,

$$
\left(\frac{\eta(\mathfrak{m})}{\eta(\mathcal{O})}\right)^{2} \in F^{*} .
$$

Proof. Define $\theta=t-\bar{\omega}$ and $N=\mathbb{N}(\theta)=t^{2}-t+(1+D) / 4$. Let $g(z)=\eta(z / m) / \eta(z)$ and $f(z)=g(z)^{2}$. If one uses the basis $\mathcal{O}=[\omega-t, 1]$,

$$
\left(\frac{\eta(\mathfrak{m})}{\eta(\mathcal{O})}\right)^{2}=e_{12}((m-1)(2-t)) f(\theta) .
$$

Recall from the last section that $(\Delta(\overline{\mathfrak{m}}) / \Delta(\mathcal{O}))^{2}$ is a 24 th power in $F^{*}$. It is also well-known that $\Delta(\overline{\mathfrak{m}}) / \Delta(\mathcal{O})$ is a square in $F^{*}$ (see $[2$, p. 238]). Since $w_{F}=2$, it follows that some twelfth root $\delta$ of $\Delta(\overline{\mathfrak{m}}) / \Delta(\mathcal{O})$ is in $F^{*}$. If we now let $\alpha=\delta m$, it is an immediate consequence of the definition of $\Delta$ that for some integer $l$,

$$
\alpha=e_{12}(l) f(\theta) .
$$

Since $\pm \alpha \in F^{*}$, to prove the theorem it suffices to prove that

$$
2 l \equiv 2(m-1)(2-t)(\bmod 12) .
$$

We do this by computing the action on $\alpha$ of the Frobenius automorphism of some principal prime ideal of norm $\equiv 11(\bmod 12)$, using [5, Theorem 3]. We can always find an integer $\kappa_{0}=r_{0}+s_{0} \theta$ with $s_{0} \neq 0$ such that $\mathbb{N}\left(\kappa_{0}\right) \equiv 11$ $(\bmod 12)$. There are then infinitely many $\kappa=r+s \theta$ such that $(r, s) \equiv\left(r_{0}, s_{0}\right)$ $(\bmod 12)$ and $(\kappa)$ is a prime ideal of norm $q \equiv 11(\bmod 12)$ so we may even choose $q$ to be prime to $m$. Some appropriate choices of $r, s(\bmod 12)$ for 
each equivalence class of $D$ modulo 48 are given in the following table.

\begin{tabular}{|c|c|c|}
\hline$D(\bmod 48)$ & $r$ & $s$ \\
\hline 7 & $3-2 t$ & 2 \\
\hline 11 & $8-t$ & 1 \\
\hline 19 & $10-t$ & 1 \\
\hline 23 & $1-2 t$ & 2 \\
\hline 31 & $3-2 t$ & 2 \\
\hline 35 & $11-t$ & 1 \\
\hline 43 & $-t$ & 1 \\
\hline 47 & $1-2 t$ & 2 \\
\hline
\end{tabular}

According to [5, Theorem 3],

$$
\alpha^{\sigma((\kappa))}=e_{12}(l q)\left(f \circ q B^{-1}\right)(\theta),
$$

where

$$
\begin{gathered}
B=\left(\begin{array}{cc}
r & s N \\
-s & r+s(2 t-1)
\end{array}\right), \\
q B^{-1}=\left(\begin{array}{cc}
r+s(2 t-1) & -s N \\
s & r
\end{array}\right) \\
\equiv\left(\begin{array}{ll}
1 & 0 \\
0 & q
\end{array}\right)\left(\begin{array}{cc}
r+s(2 t-1) & -s N \\
s q^{*} & r q^{*}
\end{array}\right) \quad(\bmod 24 m),
\end{gathered}
$$

where $q^{*}$ is any integer satisfying $q q^{*} \equiv 1(\bmod 24 m)$. Let $\Gamma^{0}(m)$ be the group of integral $2 \times 2$ matrices $\left(\begin{array}{ll}a & b \\ c & d\end{array}\right)$ that satisfy $b \equiv 0(\bmod m)$, and $a d \equiv 1(\bmod m)$.

Since $f$ has rational Fourier coefficients and its 12th power is invariant under $\Gamma^{0}(m)$,

$$
\left(f \circ q B^{-1}\right)(z)=(f \circ A)(z)
$$

where we have let

$$
A=\left(\begin{array}{cc}
r+s(2 t-1) & -s N \\
s q^{*} & r q^{*}
\end{array}\right) .
$$

Lemma 8.2. If $U=\left(\begin{array}{ll}a & b \\ c & d\end{array}\right) \in \Gamma^{0}(m)$, then

$$
(g \circ U)(z)=\varepsilon e_{24}(j) g(z),
$$

and

$$
(f \circ U)(z)=e_{12}(j) f(z),
$$


where

$$
\varepsilon=\left\{\begin{array}{l}
\left(\frac{d}{c}\right)\left(\frac{d}{c m}\right) \text { if } c \text { is odd and positive, } \\
\left(\frac{c}{d}\right)\left(\frac{c m}{d}\right) \text { if } d \text { is odd and positive, }
\end{array}\right.
$$

$\left((\div)\right.$ denotes the Jacobi symbol with $\left.\left(\frac{0}{1}\right)=1\right)$, and the value of $j=j_{U}$ modulo 24 is given by:

$$
j= \begin{cases}(m-1)\left[c(d+a-3)-b m d\left(m c^{2}+1\right)\right] & \text { if } c \text { is odd and positive }, \\ (m-1)\left[-d(c+b m)-\left(d^{2}-1\right) a c\right] & \text { if } d \text { is odd and positive. }\end{cases}
$$

Proof. Let $U^{\prime}=\left(\begin{array}{cc}a & b / m \\ c m & d\end{array}\right)$. Then

$$
(g \circ U)(z)=\frac{\eta\left(U^{\prime}\left(\begin{array}{cc}
1 & 0 \\
0 & m
\end{array}\right) \circ z\right)}{\eta(U \circ z)}=\frac{\xi\left(U^{\prime}, z / m\right)}{\xi(U, z)} g(z),
$$

where $\xi(Y, z)=\eta(Y \circ z) / \eta(z)$ for an integral matrix $Y=\left(\begin{array}{ll}y_{1} & y_{2} \\ y_{3} & y_{4}\end{array}\right)$ of determinant 1 is given explicitly by Weber [8, p. 126]:

$$
\xi(Y, z)= \begin{cases}\left(\frac{y_{4}}{y_{3}}\right) e_{24}\left(v_{1}\right) \sqrt{e_{4}(3)\left(y_{3} z+y_{4}\right)} & \text { if } y_{3} \text { is odd and positive } \\ \left(\frac{y_{3}}{y_{4}}\right) e_{24}\left(v_{2}\right) \sqrt{y_{3} z+y_{4}} & \text { if } y_{4} \text { is odd and positive }\end{cases}
$$

where

$$
\begin{aligned}
& v_{1}=3+y_{3}\left(y_{4}+y_{1}-3\right)-\left(y_{3}^{2}-1\right) y_{2} y_{4}, \\
& v_{2}=-3+y_{4}\left(y_{2}-y_{3}+3\right)+\left(y_{4}^{2}-1\right) y_{1} y_{3} .
\end{aligned}
$$

The square root here is the usual branch with non-negative real part. The lemma follows easily.

Since $\alpha \in F$, we know that $\alpha=\alpha^{\sigma((\kappa))}$. Therefore,

$$
e_{12}(l) f(\theta)=e_{12}(l q) e_{12}(j) f(\theta),
$$

where $j=j_{A}$ is given by the lemma. It then follows that

$$
2 l \equiv j(\bmod 12)
$$

since $q \equiv 11(\bmod 12)$. We see from $(23)$ that the theorem will follow once we establish

$$
j \equiv 2(m-1)(2-t)(\bmod 12) .
$$

Now using the values of $r, s$ in the above table, we compute $j$ from the lemma and verify (24) in each case. Note that since the value of $j$ is only determined modulo 12 , we may replace the $q^{*}$ in $A$ with $q$ when we compute 
$j_{A}$. First, consider the four cases where $s=1$ :

$$
\begin{aligned}
j & \equiv(m-1)[-(-r+r+2 t-1-3)]+(m-1)[-b m d(m+1)](\bmod 12) \\
& \equiv(m-1)(4-2 t)(\bmod 12),
\end{aligned}
$$

the second congruence following from the useful fact that $m^{2} \equiv 1(\bmod 24)$. When $s=2$,

$$
\begin{aligned}
j & \equiv 2(m-1)\left[2-4 t-2 r-m N r-2 r^{2}+4 t r^{2}+r^{3}\right](\bmod 12) \\
& \equiv 2(m-1)(2-t)+2(m-1)\left[r^{3}+(4 t-2) r^{2}-r(m N+2)\right](\bmod 12) .
\end{aligned}
$$

In all cases, the second term in the above sum is congruent to 0 modulo 12 because

$$
\begin{aligned}
r^{3}+(4 t-2) r^{2}-r(m N+2) & \\
& \equiv \begin{cases}(m+1)\left(2 t^{3}+t^{2}+t\right)(\bmod 3) & \text { if } D \equiv 7(\bmod 24), \\
(m+1)\left(2 t^{3}+t\right)(\bmod 3) & \text { if } D \equiv 23(\bmod 24) .\end{cases}
\end{aligned}
$$

This completes the proof of the theorem.

Remark. It follows easily from Lemma 5.1 that $(\eta(\mathfrak{m}) / \eta(\mathcal{O}))^{2}$ is a generator of the ideal $\mathfrak{m} \mathcal{O}_{F}$.

DeFinition. With $\mathfrak{m}$ as above, set

$$
\bar{\eta}(\mathfrak{m})=e_{24}(m(t-2)) \eta\left(\frac{\omega-t}{m}\right) .
$$

Since $\overline{\eta(z)}=\eta(-\bar{z})$,

$$
\overline{\eta(\mathfrak{m})}=\bar{\eta}(\mathfrak{m}) .
$$

Therefore, $(\bar{\eta}(\mathfrak{m}) / \bar{\eta}(\mathcal{O}))^{2}$ is a 12 th root of $m^{12} \Delta(\mathfrak{m}) / \Delta(\mathcal{O})$ in $F$ and generates $\overline{\mathfrak{m}}$.

We conclude with a more explicit version of Theorem 7.4, for the proof of which we will need

LEMMA 8.3. Let $\mathfrak{q}$ and $\mathfrak{m}$ (of norm $q$ and $m$, respectively) be primitive integral ideals of $k$ prime to $6 D$. Let $\sigma(\mathfrak{q})$ denote the Frobenius automorphism of $\mathfrak{q}$ in $G a \ell\left(k^{a b} / k\right)$. Then

$$
\left(\frac{\eta(\mathfrak{m})}{\eta(\mathcal{O})}\right)^{\sigma(\mathfrak{q})}=\frac{\eta(\mathfrak{m} \mathfrak{q})}{\eta(\mathfrak{q})}, \quad\left(\frac{\bar{\eta}(\mathfrak{m})}{\bar{\eta}(\mathcal{O})}\right)^{\sigma(\mathfrak{q})}=\frac{\bar{\eta}(\mathfrak{m} \overline{\mathfrak{q}})}{\bar{\eta}(\overline{\mathfrak{q}})}
$$

Proof. This is another exercise in the use of the reciprocity law [5, Theorem 3]. Of course, the two formulations are equivalent. Let us use the first one. Since $\sigma$ is multiplicative, we may assume without loss of generality that $\mathfrak{q}$ is a prime ideal. Choose a basis of the form $[\omega-t, m q]$ for $\mathfrak{m} \mathfrak{q}$ so that $[\omega-t, m]$ and $[\omega-t, q]$ are bases for $\mathfrak{m}$ and $\mathfrak{q}$, respectively. Let $\theta=t-\bar{\omega}$ 
and recall that $g(z)=\eta(z / m) / \eta(z)$. Then

$$
\begin{aligned}
\frac{\eta(\mathfrak{m} \mathfrak{q})}{\eta(\mathfrak{q})} & =e_{24}[q(2-t)(m-1)] g(\theta / q), \\
\frac{\eta(\mathfrak{m})}{\eta(\mathcal{O})} & =e_{24}[(2-t)(m-1)] g(\theta) .
\end{aligned}
$$

When we apply the reciprocity law, we find

$$
\left(\frac{\eta(\mathfrak{m})}{\eta(\mathcal{O})}\right)^{\sigma(\mathfrak{q})}=e_{24}[q(2-t)(m-1)]\left(g \circ q B^{-1}\right)(B \circ \theta)
$$

with

Writing

$$
B=\left(\begin{array}{ll}
1 & 0 \\
0 & q
\end{array}\right), \quad q B^{-1}=\left(\begin{array}{cc}
q & 0 \\
0 & 1
\end{array}\right)
$$

$$
q B^{-1} \equiv\left(\begin{array}{cc}
1 & 0 \\
0 & q
\end{array}\right)\left(\begin{array}{cc}
q & 0 \\
0 & q^{*}
\end{array}\right) \quad(\bmod 24 m),
$$

we note once again that $\left(\begin{array}{ll}1 & 0 \\ 0 & q\end{array}\right)$ acts trivially on $g(z)$ and in fact so does $\left(\begin{array}{cc}q & 0 \\ 0 & q^{*}\end{array}\right)$, as is easily seen from Lemma $8.2\left(\right.$ using $\left.q^{*} \equiv q(\bmod 24)\right)$. In conclusion,

$$
\left(g \circ q B^{-1}\right)(z)=g(z) .
$$

The lemma follows since $B \circ \theta=\theta / q$.

Theorem 8.4. Suppose $k$ is a complex quadratic field of discriminant $-D$ prime to 6 . Denote the Hilbert class field of $k$ by $F$. Let $K$ be a cyclic unramified extension of degree $n$ over $k$. Let $h$ and $H$ be the class numbers of $k$ and $K$, respectively. Let $\sigma$ be as in Lemma 8.3. Choose an unramified prime ideal $\mathfrak{p}$ in $k$ of degree one and norm $p>3$ such that the image of $\sigma(\mathfrak{p})$ in $\operatorname{Gal}(K / k)$ is a generator. Choose a basis $\left[\omega-t, p^{n+1}\right]$ for $\mathfrak{p}^{n+1}$. For $i=1, \ldots, n-1$, define

$$
U_{i}=e_{24}\left[(2-t) p^{i}(p-1)^{2}\right] \frac{\eta\left(\frac{\omega-t}{p^{i+1}}\right)^{2}}{\eta\left(\frac{\omega-t}{p^{i}}\right) \eta\left(\frac{\omega-t}{p^{i+2}}\right)} .
$$

Then $U_{1}, \ldots, U_{n-1}$ are conjugate units in $F$. If $u_{i}=\mathbb{N}_{F / K}\left(U_{i}\right)$, then $u_{1}, \ldots, u_{n-1} \in E_{K}=$ the group of units of $K$ and

$$
\left[E_{K}:\left\langle-1, u_{1}, \ldots, u_{n-1}\right\rangle\right]=\frac{H}{h / n} .
$$

Pro of. Let $\delta=\bar{\eta}\left(\mathfrak{p}^{i}\right) / \bar{\eta}\left(\mathfrak{p}^{i+1}\right)$ and $\sigma=\sigma(\mathfrak{p})$. By Lemma $8.3, \delta^{\sigma} / \delta=U_{i}$ so, by Theorem 8.1, $U_{i}^{2} \in F$. But $U_{i}^{2}=\left(\delta^{\sigma} / \delta^{p}\right)^{2} \cdot \delta^{2(p-1)}$ is a product of two numbers which are squares in $F$ (by Lemma 7.1 with $\widetilde{K}=F$ ), hence $U_{i} \in F$. 
The remaining conclusions follow from Theorem 7.4 and the observation that, up to a factor of $\pm 1, u_{i}$ is the unit $\varrho_{i}$ defined in the previous section. It is useful for computations to note that

$$
u_{i}=\prod_{[\mathfrak{b}] \in S} \frac{\bar{\eta}\left(\mathfrak{p}^{i+1} \mathfrak{b}\right)^{2}}{\bar{\eta}\left(\mathfrak{p}^{i} \mathfrak{b}\right) \bar{\eta}\left(\mathfrak{p}^{i+2} \mathfrak{b}\right)},
$$

where $S$ is the subgroup of $C \ell(k)$ corresponding to $K$.

\section{References}

[1] H. Hayashi, On elliptic units and class number of a certain dihedral extension of degree 2l, Acta Arith. 44 (1984), 35-45.

[2] D. Kubert and S. Lang, Modular Units, Springer, 1981.

[3] G. Robert, Concernant la relation de distribution satisfaite par la fonction $\phi$ associée à un réseau complexe, Invent. Math. 100 (1990), 231-257.

[4] C. L. Siegel, Lectures on Advanced Analytic Number Theory, Tata Institute of Fundamental Research, 1980.

[5] H. M. Stark, L-functions at $s=1$. IV. First derivatives at $s=0$, Adv. in Math. 35 (1980), 197-235.

[6] F. R. Villegas, private communication.

[7] L. Washington, Introduction to Cyclotomic Fields, Springer, 1982.

[8] H. Weber, Lehrbuch der Algebra, Vol. 3, Chelsea, 1961.

DEPARTMENT OF MATHEMATICS

MASSACHUSETTS INSTITUTE OF TECHNOLOGY

CAMBRIDGE, MASSACHUSETTS 02139

U.S.A. 